\title{
Early onset Alzheimer's disease
}

\begin{abstract}
Early-Onset Alzheimer's Disease (EOAD), while having the same degenerative nature and physiological changes as Late-Onset Alzheimer's disease (LOAD), presents quite differently in assessment and in how it impacts the diagnosed individual and their family. The initial issues generally present prior to age 60 with deficits in visuospatial processing, language production and/or executive function, typically before memory loss becomes apparent. EOADs are often associated with a delay in diagnosis likely resulting in lost productivity and quality of life, more insidious progression, as well as greater psychosocial problems related to the age of onset and more insight and depression. Patients and families face coping with a disease process that occurs much earlier in life than the more common LOAD. This results in increased emotional distress on all involved and requires coping strategies specific to addressing the family's caregiving ability and the patients functional ability.

In this case, the patient presents with several EOAD-specific problems, Primary Progressive Aphasia (PPA), Progressive Ideomotor Apraxia (PIA) and significant executive functioning deficits. Treatment efforts are focused on maintenance of quality of life and family support.
\end{abstract}

Volume 3 Issue I - 2018

\section{Lauren Lee MS, Lee Hyer}

Georgia Neurosurgical Institute, Mercer University, USA

Correspondence: Lee Hyer, Georgia Neurosurgical Institute, Mercer University Medical School, Surgery Center Building, Macon, USA, Tel +|478-30|-2600,

Email lhyer@ganeuroandspine.com

Received: January 17, 2018 | Published: February 05, 2018
Abbreviations: EOAD, early-onset alzheimer's disease; LOAD, late-onset alzheimer's disease;

PPA, primary progressive aphasia; PIA, progressive ideomotor apraxia; CPAP, continuous positive airway pressure; MoCA, montreal cognitive assessment; MINI, mini-international neuropsychiatric interview; WASI, Wechsler Abbreviated Scale of Intelligence; RBANS, Repeatable Battery for the Assessment of neuropsychological status; DRS, dementia rating scale TMT-A \& B, trail making test-A and $\mathrm{B}$; GAD-7, generalized anxiety disorder-7; GDS-SF, geriatric depression scale- short form; BDI-II, beck depression inventoryII; FAQ, functional activity questionnaire; PHQ-9, patient health quesitonnaire-9 item; MBMD, millon behavioral medicine diagnostic; PAI, personality zssessment inventory

\section{Patient background}

The patient was driven by his wife to the initial interview in June of 2016. At that time, he was 63 years old. His primary complaint was trouble with memory that went back for a short period with increasing difficulty in areas of language, motor skills and now memory. He could not follow-through with tasks. Indications were that he was very inactive at home now and having difficulty day to day with small tasks. He had gotten worse in all cognitive areas but especially language and motor tasks. He would stare into space, not knowing how to negotiate simple motor tasks. These issues persisted and increased in severity over the course of his evaluations. He has been seen twice since that time for monitoring of his condition and consultation with his family and caregivers.

The patient was born in Alabama. Indications are that he had a good life. There were no milestone disruptions. He graduated from high school and then went into the military between ages 18 and 22 in the Air Force. He subsequently worked for the Air Force as a sheet metal mechanic for roughly 40 years. He last worked two years ago but he was having problems for two years prior to that on the job. He was married at age 20 to his now wife. They have been married for 42 years and they have four children. They live locally and his wife continues to work. His children are very supportive and help out with their dad. He does have a family history of cognitive problems: Indications are that he has a family with "senile dementia."

His medical records indicated a mild history of depressive symptoms. He had been taking Prozac (Fluoxetine $\mathrm{HCl}$ ). His other medications are Aricept (donepezil) and Inderal (propranolol). Scans (fMRI and EEG) showed no abnormalities at that time. He denies current or past use of alcohol, tobacco, or illicit substances. He has only mild pains in his knees. He has difficulty walking, however. He reported no issues with sleep, despite suffering from sleep apnea for about 10 years. He reports being adherent to his Continuous Positive Airway Pressure (CPAP) regimen, which included wearing it at night to prevent obstructed air flow while sleeping. Further inquiry found that he sleeps frequently, day and night. He had not participated in a formal exercise program. In June of 2016, he was very inactive cognitively at home, doing little to challenge his thinking abilities. He also had trouble with mechanical things, such as his phone. His motor problems involved global issues; he was slow, confused about the sequence of his actions, and had action tremors. These formed into "jerks." His psychiatric history was unremarkable aside from the Prozac prescribed by his neurologist.

Mental status at the time of the initial evaluation revealed that he was casually dressed, wore a hat, and smiled a good deal, answering questions with vagueness. He had difficulty with his language and difficulty recalling specific aspects of questions asked. He also has difficulty hearing. He smiled and tended to make a joke of issues. He also got visibly nervous when asked questions. This seemed to put excessive pressure on him. In general though, he was not depressed or anxious. There was no evidence of perceptional anomalies or delusionary thinking. His insight was lacking and his judgment was poor.

\section{Methods}

Mr. X was initially assessed using The Mini-International Neuropsychiatric Interview (M.I.N.I.) at intake, in addition to the 
Montreal Cognitive Assessment (MoCA). Preliminary assessment results indicated deficits which merited further investigation and are discussed later. Further cognitive assessment included Wechsler Abbreviated Scale of Intelligence (WASI), Repeatable Battery for the Assessment of Neuropsychological Status (RBANS), Dementia Rating Scale (DRS), Trail Making Test-A and B (TMT-A \& B), and Clock Drawing. The self-report scales included the Generalized Anxiety Disorder-7 Item (GAD-7), Geriatric Depression Scale- Short Form (GDS-SF), Beck Depression Inventory-II (BDI-II), Functional Activity Questionnaire (FAQ), Patient Health Quesitonnaire-9 item (PHQ-9), Millon Behavioral Medicine Diagnostic (MBMD), and the Personality Assessment Inventory (PAI). His caregivers were involved in interview and feedback sessions and provided useful feedback regarding the difficulties at home including confusion, increased distress at night, and frequent sleeping. The assessment session was intended to be as brief as possible to reduce Mr. X's distress, while also answering the question of which cognitive function deficits were most significant in order to focus his recommendations on that which would benefit him and his caregivers. The focus of recommendations will be holistic in nature as they will address psychological wellbeing and overall quality of life, and include the family and caregivers in the conceptualization. Functional assessments will guide recommendations for the patient, as his care needs will be determined by his level of functioning.

\section{Assessment results}

Premorbidly, he was able considered average as he completed high school, was in the service, and functioned well as a mechanic. In June of 2016, his Wechsler Abbreviated Scale of Intelligence (WASI) Vocabulary was in the below average range. At present, he exhibited evidence of cognitive problems and was having problems with his memory. He was superficially facile socially and was not oriented. His Repeatable Battery for the Assessment of Neuropsychological Status (RBANS), Dementia Rating Scale (DRS), and Montreal Cognitive Assessment (MoCA) scores were very impaired with problems, mostly related to language, memory, visuospatial ability, and problemsolving. His problems with instrumental activities of daily living (IADLs) and function at home resulted in marked inactivity. He had evident problems with hearing. Questions had to be repeated. He was not in pain at the present. He has speech and word-finding problems. His effort was adequate.

\section{Cognitive and affective domain results}

His scores on measures of attention were impaired, in the $1 \mathrm{st} \%$ ile, on the RBANS. He could not do Trail Making Test-A (TMT-A). He had problems on the MoCA on attention, also. He could not repeat numbers backward. He was very poor on the DRS Attention (10\%).

Mr. X scored 50 on the RBANS Visuospatial/visuoconstructional Index $(.1 \%)$. He had difficulty with basic line orientation. He could not draw a Clock or complex designs. His DRS Construction was $40 \%$, his highest score. He has also had problems organizing his motor tasks, mild apraxia. Recall that he has an action tremor.

On the RBANS Language Index, he scored 74(4\%). He had no problems with Picture Naming. He had problems with fluency, both categorical and phonemic. He was lower on Vocabulary. His Conceptualization (DRS) was $6 \%$. His speech was a problem as he had word finding and vacancy issues.
Mr. X's memory scores were impaired overall. He scored in the impaired range on the RBANS Immediate Memory (Index $=44, .1 \%$ ), showing a poor learning curve. He had no problems with Remote Personal Memory (10/10).

On Delayed Memory, he had problems also (RBANS Index score $=40, .1 \%)$. He was able to recall $0 / 10$ words, $2 / 12$ story recall bits, and $0 / 20$ figural recall data points. He scored well below average on the recognition task (12/20). He did $0 / 5$ on MoCA. He was $<5 \%$ on DRS.

He has real problems in this area.

On executive functions (EF) he scored in the impaired range on all tasks administered including the DRS Initiation/Perseveration $(\mathrm{I} / \mathrm{P})$, fluency and abstraction, arithmetic, and the Clock Drawing. He was unable to complete the TMT-B. Additionally, he endorsed such difficulty on the self-report Functional Activities Questionnaire (FAQ). His IADLs were barely adequate. He did not handle money or meds, though was still driving. He slept quite frequently, day and night. He denied significant pain, giving a 2/10 rating.

Emotionally, he endorsed symptoms and received scores indicative of anxiety on the MINI. The self-report items were read to him. On the Generalized Anxiety Disorder-7 Item (GAD-7) he was anxious and irritable. His scores on the Geriatric Depression Scale- Short Form (GDS-SF), Beck Depression Inventory-II (BDI-II), and the Patient Health Quesitonnaire-9 item (PHQ-9) indicate depression. He endorsed depression and anxiety on the Millon Behavioral Medicine Diagnostic (MBMD) as well as the Personality Assessment Inventory (PAI), both of which produced valid profiles. The MBMD indicated that he has an oppositional and denigrated personality profile. This is a personality pattern reflective of an independent male who could be moody when stressed.

Summary of assessment results concluded that he has been in a steep decline cognitively and showed problems with motor skills. His behavior is distinctive for his apathy and emotional confusion. He shows cognitive domain problems distinct for logopenia and constructional apraxia. He also has problems communicating clearly with other people because of speech that was tangential or circumstantial. His skills on visuospatial areas reflected deficits. This patient has clear difficulty with fine motor tasks, especially sequencing, and has a clear action tremor. He is low on memory and attention as well as new learning. He does not have sufficient skills to handle some needed IADL tasks. He leads a simple life and has support. Affectively, he is mildly depressed. He is passive and moody. He seems to worry and get irritated. His thought processes is marked by confusion, distractibility, and difficulty concentrating.

Sleep is a problem as he sleeps excessively. He has mild pain. He is unable to do simple motor tasks and has been slowing down. He is also clearly cognitively impaired and this combination makes for poor cortical and subcortical deficiencies.

\section{Follow-up visits}

On subsequent visits his dementia progressed. Indications are that he became more irritable and was sundowning. He now has several caregivers during the day, most of whom are his family. He is still in minor pain in his knee and back. He now sleeps well at night and continues to sleep excessively during the day. He sometimes gets confused at night. He does none of his IADLs and is having some 
difficulty with ADLs, especially toileting. He is having difficulty understanding things around the house.

On the last visit, his decline was minimal. Feedback reflected that he has a rapid dementia and is in the later stages. He no doubt has had this version of an EOAD for a few years and is expressing it in the form of Progressive Ideomotor Apraxia (along with other problems of an EOAD). He is quite impaired and has no ability to negotiate his life motorically or with ideation. He has no IADL capacity and a fast decreasing ADL capacity. He is reasonably docile but shows some agitation and many behavior problems (apathy, confusion, stubbornness). He is well cared for and his children and wife have made plans for his present and future. He also has the care possibility of a caregiver organization in the VA. All are meaningfully involved. $\mathrm{He}$ is a candidate for a Long-Term Care facility now. The family was given feedback on his status and problems. They will make the decision about his long-term care entry.

\section{Early onset alzheimer's dementia (EOAD)}

This is a more common disorder than typically thought. Diagnoses make up approximately $4-10 \%$ of Alzheimer's related diagnoses and presents itself somewhat differently than the widely known late-onset Alzheimer's dementia (LOAD). ${ }^{1}$ Diagnosis occurs prior to the age of 64 and shows itself as very unexpected and upsetting to all: relatives become caregivers "off time." Prevalence is on the rise and increasingly is diagnosed in one's 40s, 50s, and 60s. The typical markers of Alzheimer's disease (AD) including the extracellular amyloid-positive neuritic plaques and intra-cellular taupositive neurofibrillary tangles (NFTs) are present but in different concentrations and locations. This results in variants in presentation when compared to LOADs. A family history of AD is a characteristic of $60 \%$ of people with EOAD.

EOADs are often associated with a delay in diagnosis likely resulting in lost productivity and quality of life, more insidious progression, as well as greater psychosocial problems related to the age of onset and more insight and depression. This disorder is most often identified by the presence of early symptoms (see below) and then a neuropsychological evaluation. One imaging technique that may soon help with such a diagnosis is the radionuclide flourine $18 \mathrm{~F}$ called Vizamyl (flutemetamol) in combination with PET, as it identifies toxic beta-amyloid in the brain. ${ }^{2}$ There is no cure and the unfolding of the cognitive/behavioral/emotional symptoms therefore lacks an antidote. The cholinesterase inhibitors (ChEIs) are ineffective as is memantine, the other cognitive helper medications. Therefore, Behavioral and Psychological Symptoms of Dementia (BPSD) are addressed with psychiatric medications and a careful arrangement of the environment and caregiver. Current treatments generally involve a Watch and Wait protocol as well as a healthy lifestyle and a watch for behavioral management -- sleep and problems of AD (sundowning, wandering) become major problems. Caregivers are naturally central to the diagnosis and treatment. Importantly, EOAD symptoms may reflect other potential paths of psychiatric (bipolar) and medical (neurological disorders) problems, often slowing the progress of the disease and making the patient more comfortable.

\section{Symptoms}
a. Short term memory down
b. Challenges in planning, problem solving
c. Difficulty in completing familiar tasks

\section{d. Confusion with time and place}

e. Trouble with visual images

f. New problems with words

\section{g. Decreased insight}

\section{h. Changes in mood}

EOADs are being called "Type 2" Alzheimer's Disease due to a difference in presentation from LOADs and overlap among these differing presentations. Estimates of $22 \%-64 \%$ of EOAD cases are considered non-typical, or non-amnestic, with the primary deficits being visuospatial, executive and/or aphasic. ${ }^{3}$ In addition to a more rapid onset of symptoms, EOADs tend to involve posterior cortical NFTs earlier and relative sparing of the hippocampus. Surprisingly, only $5 \%$ of EOADs are considered heritable, though EOAD patients with the apolipoprotein E4 (APOE e4) allele tend to progress even more quickly. ${ }^{4}$ However, additional research into the APOEe4 allele's impact on EOAD patients indicates that the allele is more common in later onset of EOADs, as well as earlier onset of LOAD, which tends to reflect Mr. X's later onset of symptoms as well as insidious progression. ${ }^{5}$ His APO e status was, however, not determined.

The most common phenotype impacts language production, termed logopenic variant of primary progressive aphasia (lvPPA), and presents as rapid onset of progressive aphasia, difficulty with word finding, frequent pauses, phonological paraphasias, decreased span of information stores, and spared grammar and articulation. Other variants include posterior cortical atrophy (PCA) which involves visuospatial deficits, a biparietal phenotype with progressive ideomotor apraxia (PIA) and visuospatial involvement, a frontal variant which involves behavioral and dysexecutive issues, and corticobasal syndrome involving progressive limb apraxia and motor difficulties. Of interest, EOAD is often confabulated with the behavioral variant of Frontal Temporal Dementia (bvFTD), one other common cause of early-onset dementia. FTD comes in several varieties (behavior, language, visuospatial or apraxia). Emerging imaging techniques, like Multiparametric MRI, are attempting to outline structural differences and resting state differences between the two diagnoses. ${ }^{6}$

Some risk factors for dementia, including lower cardiovascular fitness in early life, lower cognitive reserve in early adulthood, and traumatic brain injury (TBI), seem to have a greater impact on these patients. Counterintuitively, these patients have fewer medical comorbidities such as diabetes and other conditions which impact vasculature. Additionally, as noted above these patients have a lower frequency of the APOEe4 allele among phenotypic variants, less hippocampal and mesial temporal lode impact, but a greater impact with neurofibrillary tangles and neuritic plaques, primarily in the posterior cortex and less so in the frontal cortex. ${ }^{3}$

This patient's presentation of EOAD fits quite well into the Type-2 diagnostic category. Mr. X's cognitive decline was insidious, with his first presentation in our office having significantly impaired scores. Specifically, Mr. X seems to exhibit an overlap of phenotypic variants of EOADs. His significant aphasic and language difficulties represent the lvPPA phenotype, while his visuospatial and motor difficulties are reminiscent of the PCA and PIA phenotypes, respectively. Additionally, he exhibits behavioral symptoms and executive function deficits like that of the frontal variant. His diagnosis was on the first presentation to the clinic but shows the rapid decline. He lacks the common comorbid diagnosis of vascular issues and diabetes. The 
status of brain changes on imaging is inconclusive. It is also not known whether he exhibits the common biomarkers of EOAD. More in-depth history would indicate which symptoms presented first, though it seemed that treatment was sought when memory became impacted and after other issues were present.

Ideally, diagnosis would occur at the earliest stages of decline and functional assessment would guide treatment decisions. ${ }^{7}$ In $\mathrm{Mr}$. X's case, he may have been able to have more quality of life over the course of his disease. When he first presented, he could still read and handle many of life's tasks. By providing him with more insight into what was happening and more accommodation for his difficulties in the home he may have maintained more autonomy, reducing the caregiving load on his family. Instead, his family was presented with news that required immediate changes in the way they lived their lives. They became his caregivers around the clock.

Despite the presentation and time of diagnosis, the focus remains on quality of remaining life. In this case, his trajectory of decline was precipitous. The diagnosis was late and then the goal became educating and preparing the family for what was to come. The necessity for nursing care as the disease progresses seems to be the same for EOAD and LOAD. ${ }^{7}$ In his later visits, Mr. X was present physically, despite his slow and cautious gate and difficulty ambulating, but mostly absent otherwise. He was difficult to converse with, something his family is dealing with regularly. His family has adapted to his condition quite well, sharing responsibilities, understanding the reason for his behavior, and attempting to give him as much quality as possible. They have regimented his day and organized his constant care. As his condition progresses they are considering long-term care, understanding their limitations. As clinicians, the family becomes the patient as much as $\mathrm{Mr}$. $\mathrm{X}$ as their quality of life determines his remaining quality of life.

This case report attempts to illuminate a disease process that has not received as much attention as its more common LOAD. While diagnosis of neurodegenerative disease is always terrifying, EOAD patients and families are faced with an even faster progression at a time in life where they are normally preparing for retirement, not nursing care. This case presents a possible format of care for future families facing the same diagnosis. It also hopes to inform future research into EOAD diagnoses and inform clinicians of the prevalence of this presentation. It is possible that earlier neuropsychological assessment would have been able to detect changes in areas of visuospatial, executive, and language functioning while also noticing gait and ambulatory changes indicative of apraxia. Clinical interview which reveals family history of "senile dementia" further indicates consideration of a neurodegenerative process. Therefore, this case report hopes to provide one clear representation of a Type- 2 AD to inform clinical practice. ${ }^{8}$

\section{Acknowledgements}

The authors would like to thank the patient and family who took part in this case study.

\section{Conflict of interest}

The authors report no potential for conflict of interest in this case. The authors alone are responsible for the content and writing of the paper.

\section{References}

1. Cacace R, Sleegers K, Broeckhoven VC. Review Article: Molecular genetics of early-onset Alzheimer's disease revisited. Alzheimers Dement. 2016;12(6):733-748.

2. Gabriel M, Vernooij RWM, Fuentes Padilla P, et al. 18F PET with flutemetamol for the early diagnosis of Alzheimer's disease dementia and other dementias in people with mild cognitive impairment (MCI). Cochrane Database of Systematic Reviews. 2017;22(11).

3. Mendez MF. Early-Onset Alzheimer Disease. Neurologic Clinics. 2017;35(2):263-281

4. Tellechea P, Pujol N, Esteve BP, et al. Review article: Early- and lateonset Alzheimer disease: Are they the same entity? Neurología. 2015;S0 213-4853(15):00210-00218.

5. De Luca V, Orfei MD, Gaudenzi S, et al. Inverse effect of the APOE epsilon4 allele in late- and early-onset Alzheimer's disease. Eur Arch Psychiatry Clin Neurosci. 2016;266(7):599-606.

6. Canu E, Agosta F, Mandic SG, et al. Multiparametric MRI to distinguish early onset Alzheimer's disease and behavioural variant of frontotemporal dementia. Neuroimage Clin. 2017;15:428-438.

7. Wattmo C, Wallin ÅK. Early- versus late-onset Alzheimer disease: Longterm functional outcomes, nursing home placement, and risk factors for rate of progression. Dementia and Geriatric Cognitive Disorders Extra. 2017;7(1):172-187.

8. Ossenkoppele R, Strandberg O, Palmqvist S, et al. Distinct 18F-AV-1451 tau PET retention patterns in early- and late-onset Alzheimer's disease. Brain. 2017;140(1):2286-2294. 